\title{
DERIVADOS CITOTÓXICOS DE VITANOLIDOS ISOLADOS DAS FOLHAS DE Acnistus arborescens
}

\section{Sandro Minguzzi*}

Curso de Química, Universidade Estadual de Mato Grosso do Sul, Unidade de Naviraí, Rua Emílio Máscoli, 275, 79950-000

Naviraí - MS, Brasil

Lauro E. S. Barata

Instituto de Química, Universidade Estadual de Campinas, CP 6154, 13083-970 Campinas - SP, Brasil

Geoffrey A. Cordell

Natural Products Inc., 60203 Evanston - IL, USA

Recebido em 8/3/10; aceito em 10/9/10; publicado na web em 7/1/11

\begin{abstract}
CYTOTOXIC DERIVATIVES OF WITHANOLIDES ISOLATED FROM THE LEAVES OF Acnistus arborescens.. In view of anticancer activity of $7 \beta$-acetoxywithanolide D (2) and 7 $\beta$-16 $\alpha$-diacetoxywithonide D (3), isolated from the leaves of Acnistus arborescens (Solanaceae), five withanolide derivatives were obtained and their structures were determined by NMR, MS and IV data analysis. The in vitro anticancer activity of these derivatives was evaluated in a panel of cancer cell lines: human breast (BC-1), human lung (Lu1), human colon (Col2) and human oral epidermoid carcinoma (KB). Compounds 2a (acetylation of 2), 3b (oxidation of $\mathbf{3}$ ) and $\mathbf{2 c}$ (hydrogenation of 2) exhibited the highest anticancer activity against human lung cancer cells, with $\mathrm{ED}_{50}$ values of 0.19 , 0.25 and $0.63 \mu \mathrm{g} / \mathrm{mL}$, respectively.
\end{abstract}

Keywords: Acnistus arborescens; withanolides derivatives; human lung cancer.

\section{INTRODUÇÃO}

As plantas superiores constituem uma das fontes mais importantes de novas substâncias utilizadas diretamente como agentes medicinais; além disso, fornecem modelos para modificações estruturais e organização de propriedades farmacológicas e bioquímicas, servindo de inspiração para químicos orgânicos na construção de novos arranjos moleculares naturais. ${ }^{1}$ Dentro do grupo dos esteroides naturais com atividades citotóxicas se encontram os vitanolidos, que formam o grupo mais abundante de compostos que possuem uma $\delta$ lactona em sua cadeia lateral. Estes foram primeiramente citados na literatura em 1962, mas sua elucidação estrutural só aconteceu em 1966 com a descrição estereoquímica da vitaferina 1 (Figura 1) isolada das folhas de Withania somnifera, popularmente conhecida na Índia como Ashwaganha. ${ }^{2}$ Os vitanolidos têm sido isolados de plantas de vários gêneros, como exemplo podem ser citados: Acnistus, ${ }^{3-8}$ Datura, ${ }^{9}$ Jaborosa,${ }^{10}$ Physali ${ }^{11} \mathrm{e}$ Withania, ${ }^{12,13}$ todos da família Solanaceae, e despertam interesse por apresentarem diversas atividades biológicas, algumas delas como antitumoral, ${ }^{4-8}$ diurética, ${ }^{13}$ indução de quinona redutase ${ }^{5,14}$ e imunossupressora. ${ }^{15}$

Outros esteroides que são encontrados na famíla Solanaceae incluem glicosídeos e alcaloides esteroidais, compostos presentes nos gêneros Cestrum e Solanum, sendo este último gênero o que possui o maior número de espécies catalogadas. ${ }^{16,17}$ Os primeiros trabalhos sobre a atividade farmacológica de vitanolidos em Acnistus arborescens foram feitos sobre inibidores de tumores; em um deles, utilizando-se do extrato alcoólico das folhas, observou-se significativa atividade biológica in vitro contra células de carcinoma humano da nasofaringe e in vivo contra sarcoma $180 \mathrm{em}$ ratos; a ação farmacológica foi atribuída à vitaferina 1 1. $^{3}$

*e-mail: sming@uems.br

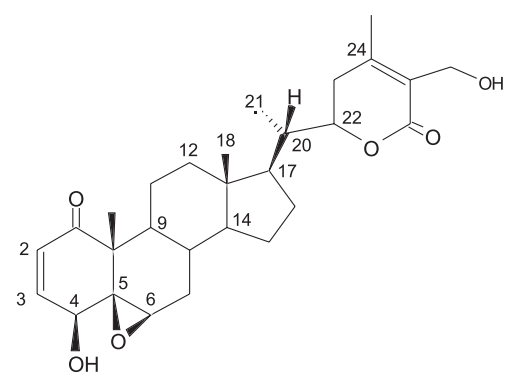

Figura 1. Estrutura da vitaferina 1

Acnistus arborescens conhecida popularmente como marianeira ou esporão de galo falso apresenta-se distribuída pelas Américas do Sul e Central; nesta espécie têm sido relatados vários compostos da classe dos vitanolidos incluindo vitacnistinas, ${ }^{3}$ vitafisalinas ${ }^{6,7}$ e derivados do vitanolido D: $12 \beta$-acetoxi-4-deoxi-5,6-deoxi- $\Delta^{5}$ vitanolido $\mathrm{D},{ }^{8} 7 \beta$-acetoxivitanolido D 2, 7 $\beta, 16 \alpha$-diacetoxivitanolido D 3 e 4-deoxi-7 $\beta, 16 \alpha$-diacetoxivitanolido D. ${ }^{5}$ A atividade citotóxica de alguns destes compostos foi verificada contra células tumorais in vitro, destacando-se as substâncias $\mathbf{2}$ e $\mathbf{3}$ contra células de cólon (LNCaP) com $\mathrm{ED}_{50}$ de 0,03 e $0,08 \mu \mathrm{g} / \mathrm{mL}$, respectivamente. ${ }^{5}$

Levando-se em conta a potencial atividade anticâncer dos vitanolidos, este trabalho se focou na obtenção de cinco derivados a partir dos vitanolidos $\mathbf{2}$ e $\mathbf{3}$, com a realização de testes de citotoxicidade em células cancerosas de maior incidência em humanos.

\section{PARTE EXPERIMENTAL}

\section{Aspectos gerais}

Os espectros de RMN foram registrados em equipamento Varian Inova-500 (500 MHz para ${ }^{1} \mathrm{H}$ e $125 \mathrm{MHz}$ para $\left.{ }^{13} \mathrm{C}\right)$, utilizando-se o 
sinal do solvente como referencial interno. Os espectros de massa foram obtidos através de injeção direta em espectrômetro VG Autoespec High Resolution Mass Spectrometer (Micromass Company). Os espectros de infravermelho (IV) foram obtidos na forma de filmes em espectrofotômetro Perkin Elmer 1600 (FTIR Series). Nas separações por cromatografia em coluna foi utilizada sílica gel 70-230 da Merck. As frações foram monitoradas por cromatografia em camada delgada (CCD) em cromatoplacas PF254 (Merck) e as manchas com vitanolidos foram reveladas com revelador ácido sulfúrico/MeOH (1:1) ou com irradiação no UV a $254 \mathrm{~nm}$.

\section{Isolamento dos constituintes}

As folhas de Acnistus arborescens (1,3 kg) foram secas, moídas e submetidas à extração através de maceração com etanol $(5 \mathrm{~L})$. Após evaporação do solvente procedeu-se à partição entre acetato de etila/ EtOH: $\mathrm{H}_{2} \mathrm{O}$ (6:4). A fração acetato de etila foi filtrada em Celite e após evaporação, fracionada em coluna cromatográfica empacotada com sílica gel 70-230 da Merck e eluída com misturas de hexano/ AcOEt. Foram recolhidas 74 frações de $50 \mathrm{~mL}$ que foram agrupadas posteriormente em 16 frações, através de monitoramento por CCD. A fração 36-40 eluída com hexano:AcOEt (30:70) foi submetida repetidas vezes à separação em cromatografia em camada delgada preparativa (CCDP), utilizando-se hexano:AcOEt (20:80) como eluente e obtendo-se 2 ( $1,0 \mathrm{~g}$ ) com $0,07 \%$ de rendimento. O composto 3 (300 mg) foi obtido por CCDP usando a fração 40-55 eluída com hexano:AcOEt (30:70), seguida de separação em CCDP, obtendo-se um rendimento de $0,02 \%$ desta substância nas folhas.

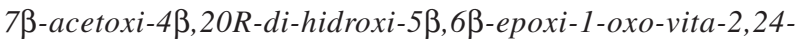
dienolido (2). Cristais incolores e inodoros. P.F. $=151-153^{\circ} \mathrm{C}$. Dados espectrométricos similares aos da literatura. ${ }^{4}$

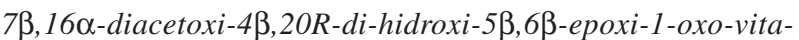
2,24-dienolido (3). Cristais incolores e inodoros. P.F. $=163-166{ }^{\circ} \mathrm{C}$. Dados espectrométricos similares aos da literatura. ${ }^{5}$

\section{Obtenção dos derivados}

\section{Acetilação de 2 e $\mathbf{3}$}

O vitanolido $2(20 \mathrm{mg})$ foi dissolvido em $2 \mathrm{~mL}$ de acetato de etila e, então, adicionado a um balão de $50 \mathrm{~mL}$, adicionando-se $1 \mathrm{~mL}$ de anidrido acético-piridina (1:1), iniciando-se agitação e deixando-se reagir por $3 \mathrm{~h}$, sendo monitorada a formação do produto por $\mathrm{CCD}$, obtendo-se $18 \mathrm{mg}$ de $\mathbf{2 a}$, com rendimento de $83 \%$. O mesmo procedimento foi adotado para o vitanolido 3, obtendo-se $17 \mathrm{mg}$ de $\mathbf{3 a}$, com rendimento de $85 \%$.

$4 \beta, 7 \beta$-diacetoxi-20R-hidroxi-5 $\beta$, $6 \beta$-epoxi-1-oxo-vita-2,24dienolido (2a). Óleo incolor e inodoro. $\lambda$ max. $\mathrm{cm}^{-1}$ : 3440, 1721, 1703, 1682. EIMS (intensidade relativa): 586 ( $\mathrm{M}^{+}$, não detectado), $461[\mathrm{M}-125]^{+}(13), 401[\mathrm{M}-125-\mathrm{HOAc}]^{+}(75), 341[\mathrm{M}-125-2 \mathrm{HOAc}]^{+}$ (25), $169\left[\mathrm{C}_{9} \mathrm{H}_{13} \mathrm{O}_{3}\right]^{+}(40), 126\left[\mathrm{C}_{7} \mathrm{H}_{10} \mathrm{O}_{2}\right]^{+}(100) . \mathrm{RMN}{ }^{1} \mathrm{H}[500 \mathrm{MHz}$, (ppm), $\mathrm{CDCl}_{3}$ ]: 6,25 (d, 9,8 Hz, H-2), 7,00 (dd, 5,5 e 9,8 Hz, H-3), 4,65 (d, 5,5 Hz, H-4), 3,35 (d, 1,9 Hz, H-6), 4,85 (dd, 1,9 e 9,3 Hz, $\mathrm{H}-7), 4,15$ (dd, 3,2 e 12,5 Hz, H-22), RMN ${ }^{13} \mathrm{C}[125 \mathrm{MHz},(\mathrm{ppm})$, $\mathrm{CDCl}_{3}$ ]: 201,0 (C-1), 134,0 (C-2), 140,0 (C-3), 71,0 (C-4), 64,0 (C5), 60,5 (C-6), 74,5 (C-7), 34,0 (C-8), 44,0 (C-9), 48,0 (C-10), 21,8 (C-11), 39,0 (C-12), 44,0 (C-13), 55,5 (C-14), 25,4 (C-15), 28,0 (C16), 53,8 (C-17), 13,3 (C-18), 15,5 (C-19), 75,2 (C-20), 20,5 (C-21), 80,7 (C-22), 31,8 (C-23), 148,2 (C-24), 122,0 (C-25), 166,0 (C-26), 12,4 (C-27), 20,7 (C-28), 169,0 (4-OAc- C=O), 20,6 (4-OAc- $\left.\mathrm{CH}_{3}\right)$, $171,0$ (7- OAc- $\mathrm{C}=\mathrm{O}), 21,3\left(7-\mathrm{OAc}-\mathrm{CH}_{3}\right)$.

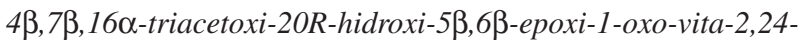
dienolido (3a). Óleo incolor e inodoro. IV $v_{\text {máx }} \mathrm{cm}^{-1}: 3440,1718$, 1709, 1682. EIMS (intensidade relativa): 644 ( $\mathrm{M}^{+}$, não detectado), $524\left[\mathrm{M}-2 \mathrm{HOAc}^{+}\right.$(32) $519[\mathrm{M}-125]^{+}(13), 501\left[\mathrm{M}-125-\mathrm{H}_{2} \mathrm{O}\right]^{+}(8)$, $399\left[\mathrm{M}-125-2 \mathrm{HOAc}^{+}(17), 169\left[\mathrm{C}_{9} \mathrm{H}_{13} \mathrm{O}_{3}\right]^{+}(35), 126\left[\mathrm{C}_{7} \mathrm{H}_{10} \mathrm{O}_{2}\right]^{+}(100)\right.$. RMN ${ }^{1} \mathrm{H}\left[500 \mathrm{MHz}, \delta(\mathrm{ppm}), \mathrm{CDCl}_{3}\right.$ ]: 6,25 (d, 10,1 Hz,H-2), 7,0 (dd, 5,7 e 10,1 Hz, H-3), 4,7 (d, 5,7 Hz, H-4), 3,4 (d, 1,8 Hz, H-6), 4,85 (dd, 1,8 e 9,3 Hz, H-7), 4,2 (dd, 3,6 e 13,0 Hz, H-22), 5,35 (m, H-16), RMN ${ }^{13} \mathrm{C}\left[125 \mathrm{MHz},(\mathrm{ppm}), \mathrm{CDCl}_{3}\right]$ : 201,0 (C-1), 134,0 (C-2), 140,1 (C-3), 71,0 (C-4), 64,0 (C-5), 60,8 (C-6), 74,5 (C-7), 33,5 (C-8), 43,0 (C-9), 47,5 (C-10), 21,0 (C-11), 40,0 (C-12), 43,5 (C-13), 53,0 (C-14), 34,0 (C-15), 75,5 (C-16), 59,5 (C-17), 14,7 (C-18), 16,0 (C-19), 75,6 (C-20), 20,9 (C-21), 81,0 (C-22), 30,5 (C-23), 149,0 (C-24), 122,5 (C-25), 166,0 (C-26), 12,5 (C-27), 20,7 (C-28), 170,0 (4-OAc- C=O), 21,0 (4-OAc- $\mathrm{CH}_{3}$ ), 170,5 (7- OAc- $\left.\mathrm{C}=\mathrm{O}\right), 21,6\left(7-\mathrm{OAc}^{-} \mathrm{CH}_{3}\right.$ ), 171,4 (16- OAc- $\mathrm{C}=\mathrm{O}), 21,2\left(16-\mathrm{OAc}-\mathrm{CH}_{3}\right)$.

\section{Oxidação de 2 e 3}

Inicialmente foi necessária a obtenção de óxido de manganês $\left(\mathrm{MnO}_{2}\right)$ ativado, uma vez que o óxido de manganês comercial não é adequado para síntese. Preparou-se uma solução contendo $5 \mathrm{~g}$ de $\mathrm{KMnO}_{4}$ em $10 \mathrm{~mL}$ de água, que foi adicionada a uma solução contendo $5 \mathrm{~g}$ de $\mathrm{MnSO}_{4}$ em $20 \mathrm{~mL}$ de água, numa temperatura de $90{ }^{\circ} \mathrm{C}$. A mistura foi mantida em agitação por $20 \mathrm{~min}$, seguindo-se então a filtração do óxido de manganês (composto de cor marrom avermelhada) em papel de filtro. O precipitado foi lavado com 90 $\mathrm{mL}$ de água e, em seguida, com $90 \mathrm{~mL}$ de acetona. O sólido obtido foi seco em estufa a $100{ }^{\circ} \mathrm{C}$. O óxido de manganês preparado por este método é ativo por até 1 mês, desde que guardado em frasco vedado em dessecador. ${ }^{18}$

A partir desta etapa iniciou-se a reação em um balão de $50 \mathrm{~mL}$ adicionando-se $50 \mathrm{mg}$ do vitanolido 2 dissolvido em $20 \mathrm{~mL}$ de acetona, seguido da adição de $200 \mathrm{mg}$ de $\mathrm{MnO}_{2}$; a reação foi feita sob agitação à temperatura ambiente, com acompanhamento por CCD. Após filtração, evaporação do solvente e recristalização com metanol obteve-se $20 \mathrm{mg}$ de $\mathbf{2 b}$, com $40 \%$ de rendimento. A partir de $20 \mathrm{mg}$ de 3 e seguindo-se o mesmo procedimento anterior, obteve-se $7 \mathrm{mg}$ de $3 \mathbf{b}$, com $35 \%$ de rendimento.

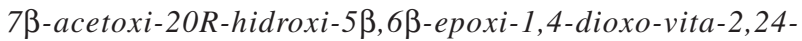
dienolido (2b). Cristais incolores, translúcidos e inodoros. P.F.= 137-140 ${ }^{\circ} \mathrm{C} . \lambda \max \mathrm{cm}^{-1}: 3435,1720,1712,1686$. EIMS (intensidade relativa): $526\left(\mathrm{M}^{+} \text {, não detectado), 466[M- HOAc }\right]^{+}(7) 401[\mathrm{M}-125]^{+}$ (13), 383[M-125- $\left.\mathrm{H}_{2} \mathrm{O}\right]^{+}(18), 169\left[\mathrm{C}_{9} \mathrm{H}_{13} \mathrm{O}_{3}\right]^{+}(26), 126\left[\mathrm{C}_{7} \mathrm{H}_{10} \mathrm{O}_{2}\right]^{+}$ (100). RMN ${ }^{1} \mathrm{H}\left[500 \mathrm{MHz}, \delta\right.$ (ppm), $\left.\mathrm{CDCl}_{3}\right]$ : 6,85 (s, H-2 e H-3), $3,45$ ( $d, 1,9 \mathrm{~Hz}, \mathrm{H}-6), 4,82$ (dd, 1,9 e 9,3 Hz, H-7), 4,13 (dd, 3,0 e $12,3 \mathrm{~Hz}, \mathrm{H}-22), \mathrm{RMN}{ }^{13} \mathrm{C}$ [125 MHz, (ppm), $\left.\mathrm{CDCl}_{3}\right]$ : 202,0 (C-1), 139,0 (C-2), 142,0 (C-3), 193,0 (C-4), 65,0 (C-5), 63,8 (C-6), 74,0 (C-7), 33,5 (C-8), 43,3 (C-9), 48,8 (C-10), 22,3 (C-11), 39,8 (C-12), 42,8 (C-13), 55,5 (C-14), 24,0 (C-15), 27,0 (C-16), 53,9 (C-17), 13,5 (C-18), 19,2 (C-19), 75,0 (C-20), 20,6 (C-21), 81,1 (C-22), 31,5 (C-23), 148,0 (C-24), 122,5 (C-25), 167,0 (C-26), 12,5 (C-27), 21,0 (C-28), 172,1 (7-OAc- C=O), 21,5 (7-OAc- $\left.\mathrm{CH}_{3}\right)$.

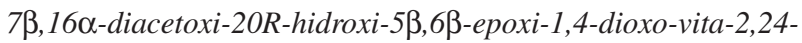
dienolido (3b). Cristais incolores, translúcidos e inodoros. P.F.= 140-143 ${ }^{\circ} \mathrm{C} . \lambda \operatorname{max~cm}{ }^{-1}: 3437,1718,1708,1682$. EIMS (intensidade relativa): $584\left(\mathrm{M}^{+}\right.$, não detectado), 524[M- $\mathrm{HOAc}^{+}(3), 459[\mathrm{M}-125]^{+}$ (25), 441[M-125- $\left.\mathrm{H}_{2} \mathrm{O}\right]^{+}$(16), $169\left[\mathrm{C}_{9} \mathrm{H}_{13} \mathrm{O}_{3}\right]^{+}(34), 126\left[\mathrm{C}_{7} \mathrm{H}_{10} \mathrm{O}_{2}\right]^{+}$ (100). RMN ${ }^{1} \mathrm{H}\left[500 \mathrm{MHz}, \delta\right.$ (ppm), $\left.\mathrm{CDCl}_{3}\right]: 6,9$ (s, H-2 e H-3), 3,6 (d, 1,9 Hz, H-6), 4,85 (dd, 1,9 e 9,3 Hz, H-7), 5,4 (t, 7Hz, H-16) 4,2 (dd, 3,4 e 12,7 Hz, H-22), RMN ${ }^{13} \mathrm{C}$ [125 MHz, (ppm), $\left.\mathrm{CDCl}_{3}\right]$ : 202,5 (C-1), 138,7 (C-2), 141,8 (C-3), 193,3 (C-4), 64,8 (C-5), 63,9 
(C-6), 74,2 (C-7), 33,6 (C-8), 43,3 (C-9), 48,8 (C-10), 22,3 (C-11), 39,8 (C-12), 42,8 (C-13), 55,5 (C-14), 24,0 (C-15), 75,0 (C-16), 53,9 (C-17), 13,5 (C-18), 19,2 (C-19), 75,0 (C-20), 20,6 (C-21), 81,1 (C-22), 31,5 (C-23), 148,0 (C-24), 122,5 (C-25), 167,2 (C-26), 12,5 (C-27), 21,0 (C-28), 170,1 (7-OAc- C=O), 21,0 (7-OAc- $\left.\mathrm{CH}_{3}\right), 171,4$ (16- OAc- $\mathrm{C}=\mathrm{O}), 21,2\left(16-\mathrm{OAc}-\mathrm{CH}_{3}\right)$.

\section{Hidrogenação de 2}

$\mathrm{O}$ processo consiste da passagem de $\mathrm{H}_{2}(\mathrm{~g})$ gerado através de reação de alumínio metálico com solução aquosa de $\mathrm{NaOH}$ a $5 \%$, na mistura contendo $50 \mathrm{mg}$ de $\mathbf{2}$ dissolvido em $10 \mathrm{~mL}$ de acetato de etila e o catalisador $\mathrm{Pd} / \mathrm{C}$ à temperatura ambiente. Após $90 \mathrm{~min}$ de reação procedeu-se à evaporação do solvente, seguida da purificação por CCDP, eluindo-se 3 vezes uma mistura de acetato/tolueno (8:2), e obtendo-se por métodos usuais de separação $30 \mathrm{mg}$ de $\mathbf{2 c}$, com $60 \%$ de rendimento.

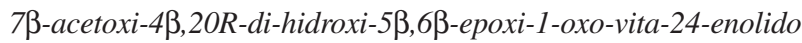
(2c). Cristais incolores, opacos e inodoros. P.F. $=140-143^{\circ} \mathrm{C} . \lambda \max$ $\mathrm{cm}^{-1}: 3430,1720,1712,1686$. EIMS (intensidade relativa): 530 $\left(\mathrm{M}^{+}\right.$, não detectado), $405[\mathrm{M}-125]^{+}(45), 387\left[\mathrm{M}-125-\mathrm{H}_{2} \mathrm{O}\right]^{+}(12)$, $169\left[\mathrm{C}_{9} \mathrm{H}_{13} \mathrm{O}_{3}\right]^{+}(20), 126\left[\mathrm{C}_{7} \mathrm{H}_{10} \mathrm{O}_{2}\right]^{+}(100)$. RMN ${ }^{1} \mathrm{H}[500 \mathrm{MHz}, \delta$ (ppm), $\mathrm{CDCl}_{3}$ ]: 3,5 (t, 3,5, H-4), 3,3 (d, 1,7 Hz, H-6), 4,9 (dd, 1,7 e 8,3 $\mathrm{Hz}, \mathrm{H}-7), 4,2$ (dd, 3,4 e 12,7 Hz, H-22), RMN ${ }^{13} \mathrm{C}[125 \mathrm{MHz}$, (ppm), $\mathrm{CDCl}_{3}$ ]: 210,0 (C-1), 31,8 (C-2), 26,5 (C-3), 72,5 (C-4), 70,0 (C-5), 57,00 (C-6), 75,8 (C-7), 34,2 (C-8), 43,0 (C-9), 49,8 (C-10), 22,8 (C-11), 39, 8 (C-12), 43,5 (C-13), 52,0 (C-14), 25,0 (C-15), 31,0 (C16), 54,0 (C-17), 15,3 (C-18), 13,5 (C-19), 76,0 (C-20), 20,8 (C-21), 81,0 (C-22), 31,8 (C-23), 148,0 (C-24), 122,0 (C-25), 167,0 (C-26), 12,4 (C-27), 20,7 (C-28), 170,1 (7-OAc- C=O), 21,0 (7-OAc- $\left.\mathrm{CH}_{3}\right)$.

\section{Avaliação do potencial citotóxico}

Os derivados obtidos (Figura 2) foram avaliados na Universidade de Illinois em Chicago, de acordo com procedimentos padrões contra BC-1 (câncer de mama), Lu1 (câncer de pulmão), Col2 (câncer de cólon), KB (carcinoma epidermoidal oral) e LNCap (câncer de próstata). ${ }^{19}$ Os resultados dos testes biológicos foram apresentados como $\mathrm{ED}_{50}$ em $\mu \mathrm{g} / \mathrm{mL}$, Tabela 1.

\section{RESULTADOS E DISCUSSÃO}

O método de extração das substâncias das folhas de Acnistus arborescens foi o da extração sucessiva a frio com etanol, seguida de partição com solventes em ordem crescente de polaridade; a partir do extrato de acetato de etila foram isolados, através de técnicas cromatográficas, os vitanolidos 2 e 3. Os vitanolidos foram identificados com base nos dados espectrais e por comparação com os dados de RMN já descritos na literatura. ${ }^{9}$ A partir do isolamento destas duas substâncias foram feitas reações para obtenção de derivados em reações de acetilação, oxidação e hidrogenação catalítica (Figura 2).

Em todos os derivados obtidos observou-se por EIMS o pico base $m / z, 126\left(\mathrm{C}_{7} \mathrm{H}_{10} \mathrm{O}_{2}\right)$ formado pela quebra da ligação C-20/C-22 com rearranjo de um $\mathrm{H}$, indicando a presença de porção $\delta$-lactona $\alpha, \beta$ insaturada; outro fragmento significativo nos derivados foi em $m / z, 169\left(\mathrm{C}_{9} \mathrm{H}_{13} \mathrm{O}_{3}\right)$ referente à clivagem da ligação C-17/C-20 que ocorre nos 20-hidroxi-vitanolidos. Nos espectros de IV dos derivados foram observadas similaridades com os precursores, com absorções em torno de 3450, 1720, 1710 e $1680 \mathrm{~cm}^{-1}$ característicos de grupamentos hidroxila, carbonila de grupo acetoxi, lactona insaturada e cetona $\alpha, \beta$ insaturada, respectivamente. ${ }^{5}$ A acetilação de $\mathbf{2}$ e $\mathbf{3}$ foi feita utilizando-se anidrido acético e piridina à temperatura ambiente, levando aos derivados $\mathbf{2 a}$ e $\mathbf{3 a}$. As reações foram acompanhadas por

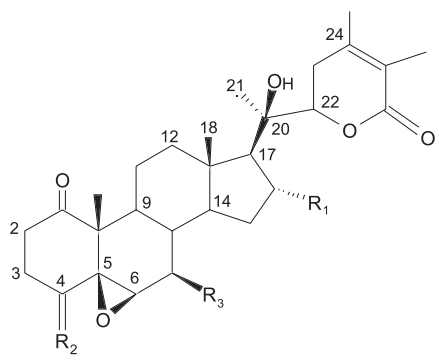

$$
\begin{aligned}
& \mathbf{2}-\Delta^{2}, \mathrm{R}_{1}=\mathrm{H}, \mathrm{R}_{2}=\beta-\mathrm{OH}, \alpha-\mathrm{H}, \mathrm{R}_{3}=\mathrm{OAc} \\
& \mathbf{3}-\Delta^{2}, \mathrm{R}_{1}=\mathrm{OAc}, \mathrm{R}_{2}=\beta-\mathrm{OH}, \alpha-\mathrm{H}, \mathrm{R}_{3}=\mathrm{OAc} \\
& \mathbf{4}-\Delta^{2}, \mathrm{R}_{1}=\mathrm{OAc}, \mathrm{R}_{2}=\mathrm{H} 2, \mathrm{R}_{3}=\mathrm{OAc} \\
& \mathbf{5}-\Delta^{2}, \mathrm{R}_{1}=\mathrm{H}, \mathrm{R}_{2}=\beta-\mathrm{OH}, \alpha-\mathrm{H}, \mathrm{R}_{3}=\mathrm{H} \\
& \mathbf{2 a}-\Delta^{2}, \mathrm{R}_{1}=\mathrm{H}, \mathrm{R}_{2}=\beta-\mathrm{OAc}, \alpha-\mathrm{H}, \mathrm{R}_{3}=\mathrm{OAc} \\
& \mathbf{2 b}-\Delta^{2}, \mathrm{R}_{1}=\mathrm{H}, \mathrm{R}_{2}=\mathrm{O}, \mathrm{R}_{3}=\mathrm{OAc} \\
& \mathbf{2 c}-\mathrm{R}_{1}=\mathrm{H}, \mathrm{R}_{2}=\beta-\mathrm{OH}, \alpha-\mathrm{H}, \mathrm{R}_{3}=\mathrm{OAc} \\
& \mathbf{3 a}-\Delta^{2}, \mathrm{R}_{1}=\mathrm{OAc}, \mathrm{R}_{2}=\mathrm{OAc}, \mathrm{R}_{3}=\mathrm{OAc} \\
& \mathbf{3 b}-\Delta^{2}, \mathrm{R}_{1}=\mathrm{OAc}, \mathrm{R}_{2}=\mathrm{O}, \mathrm{R}_{3}=\mathrm{OAc}
\end{aligned}
$$

Figura 2. Estruturas de 2, 3, 4, vitanolido $D(5)$ e dos derivados $2 a, 2 b, 2 c$, $3 a$ e $3 b$

Tabela 1. Atividade citotóxica dos compostos 2 e 3 e derivados 2a-2c, 3a e 3b

\begin{tabular}{cccccc}
\hline Compostos & \multicolumn{5}{c}{ Cultura de células $^{\mathrm{a}}$} \\
& $\mathrm{BC}-1$ & Lu1 & Col2 & KB & LNCaP \\
\hline 2 & 0,2 & 1,3 & 0,03 & 0,4 & 0,2 \\
3 & 0,4 & 2,1 & 0,08 & 0,5 & 0,2 \\
$2 \mathrm{a}$ & 1,1 & 0,19 & 0,2 & $\mathrm{nt}$ & $\mathrm{nt}$ \\
$2 \mathrm{~b}$ & $\mathrm{nt}^{\mathrm{c}}$ & 7,9 & 1,1 & 0,8 & 1,9 \\
$2 \mathrm{c}$ & $\mathrm{nt}$ & 0,63 & 3,7 & 10,6 & 13,1 \\
$3 \mathrm{a}$ & 3 & 0,25 & 0,3 & $\mathrm{nt}$ & $\mathrm{nt}$ \\
$3 \mathrm{~b}$ & $\mathrm{nt}$ & 5 & 2 & 1,7 & 0,9 \\
Taxol $^{\mathrm{b}}$ & $\mathrm{nt}$ & 0,002 & 0,004 & 0,0004 & 0,004 \\
Camptotecina $^{\mathrm{b}}$ & $\mathrm{nt}$ & 0,01 & 0,02 & 0,008 & 0,01 \\
\hline
\end{tabular}

${ }^{a}$ Os resultados são expressos como valores de $\mathrm{ED}_{50}(\mu \mathrm{g} / \mathrm{mL})$. Código para as culturas de células testadas: BC-1 = câncer de mama humano; $\mathrm{Lu} 1$ = câncer de pulmão humano; $\mathrm{Col} 2$ = câncer de cólon humano; $\mathrm{KB}=$ carcinoma oral epidermoide humano; $\mathrm{LNCaP}=$ câncer de próstata hormônio-dependente humano. ${ }^{\mathrm{b}}$ Compostos antitumorais padrão. ${ }^{21,22 \mathrm{c}} \mathrm{nt}=$ não testado

cromatografia em camada delgada (CCD) observando-se a formação dos produtos após $3 \mathrm{~h}$ de reação. Os compostos obtidos foram analisados por espectrometria de massas, RMN ${ }^{1} \mathrm{H}, \mathrm{COSY}{ }^{1} \mathrm{H}-{ }^{1} \mathrm{H}, \mathrm{RMN}$ de ${ }^{13} \mathrm{C}$, DEPT e IV. A análise do espectro de massas de $\mathbf{2 a}$ mostrou como picos importantes a $\mathrm{m} / \mathrm{z}$ 126, 169, 401 (M-125-HOAc) ${ }^{+}$e 461 $(\mathrm{M}-125)^{+}$(Figura 3). No composto $3^{\mathrm{a}}$, o fragmento a $\mathrm{m} / \mathrm{z} 519$ (M$125)^{+}$, com 58 unidades a mais que o fragmento encontrado em $\mathbf{2 a}$, mostra a presença de grupo acetoxila adicional em $\mathbf{3 a}$.

$\mathrm{O}$ espectro de $\mathrm{RMN}{ }^{1} \mathrm{H}$ de $\mathbf{2 a}$ mostrou semelhança em relação ao espectro de RMN ${ }^{1} \mathrm{H}$ de $2,{ }^{5}$ a exceção do deslocamento do $\mathrm{H}-4$ que passa de $\delta 3,8$ em 2 para $\delta$ 4,65 em 2a, desproteção esta causada pela mudança de hidroxila para um grupo acetato. No espectro de RMN ${ }^{1} \mathrm{H}$ de 3a o $\mathrm{H}-4$ aparece a $\delta$ 4,7 com uma desproteção de 0,9 ppm em relação ao H-4 em 3. ${ }^{5}$ As correlações dos hidrogênios tanto em 2a como em 3a puderam ser confirmadas através de análise dos espectros COSY ${ }^{1} \mathrm{H}-{ }^{1} \mathrm{H}$, e são destacadas na Figura 4.

Através de $\mathrm{RMN}{ }^{13} \mathrm{C}$ foi observado em $2 \mathbf{a}$ que o grupo C-4 acetoxi causa um efeito de proteção de 1,6 ppm no grupo metila em C-19 a $\delta$ 15,5 , quando comparado seu espectro com o de $2 .{ }^{5}$ Esta proteção pode 


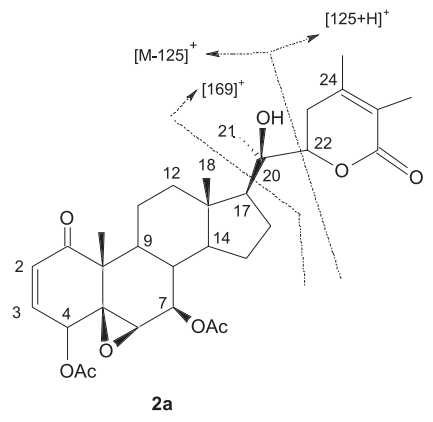

Figura 3. Fragmentação por espectrometria de massas de $\mathbf{2 a}$
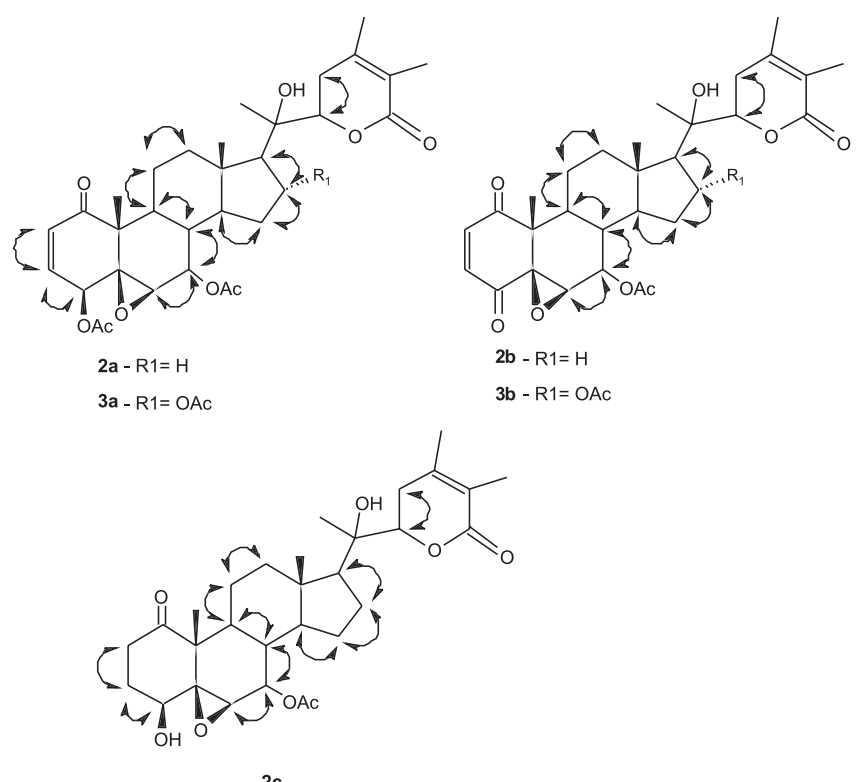

2c

Figura 4. Correlações $\operatorname{COSY}^{1} \mathrm{H}^{-1} \mathrm{H}$ observadas para $2 \boldsymbol{a}, \mathbf{2 b}, \mathbf{2 c}, \mathbf{3 a} \boldsymbol{e} \mathbf{3 b}$

ser explicada pela interação 1,3 diaxial entre C-4 acetoxi e o grupo metila em C-19. O deslocamento químico do C-4 (grupo acetoxi) em $\delta 71$ em 2 a está mais desprotegido (1,7 ppm), quando comparado com o carbono correspondente ao seu precursor $\mathbf{2}$, devido à mudança da função de hidroxila para acetoxila, este grupo $\mathrm{CH}$ a $71 \mathrm{ppm}$ pode ser observado no DEPT de $\mathbf{2 a}$; aspectos semelhantes foram observados no espectro de 3a. Nos espectros DEPT e RMN ${ }^{13} \mathrm{C}$ de $\mathbf{2} \mathbf{a}$ e $\mathbf{3} \mathbf{a}$ foram observadas adições de dois carbonos referentes à carbonila (169 e $170 \mathrm{ppm})$ e metila $(20,6$ e $21 \mathrm{ppm})$, respectivamente. As reações de oxidação de $\mathbf{2}$ e $\mathbf{3}$ foram feitas utilizando-se óxido de manganês ativado como agente oxidante. ${ }^{16} \mathrm{~A}$ confirmação das estruturas de 2a e 3a foi feita por espectrometria de massas, RMN ${ }^{1} \mathrm{H}, \mathrm{COSY}$ ${ }^{1} \mathrm{H}-{ }^{1} \mathrm{H}, \mathrm{RMN}$ de ${ }^{13} \mathrm{C}$, DEPT e IV. Na análise de RMN ${ }^{1} \mathrm{H}$ de $\mathbf{2} \mathbf{b}$ e $\mathbf{3 b}$, em comparação com $\mathbf{2}$ e 3, observam-se os desaparecimentos dos sinais dos hidrogênios vinílicos ( H-2 em 6,2 ppm (d) e H-3 em 6,9 ppm (dd)), e o aparecimento de singleto a 6,85 ppm correspondente a $2 \mathrm{H}$ vinílicos (H-2 e $\mathrm{H}-3)$ para $\mathbf{2 b}$ e um singleto em 6,9 ppm para 3b correspondendo a $2 \mathrm{H}$ vinílicos $(\mathrm{H}-2$ e $\mathrm{H}-3)$. Comparando-se a estrutura de $\mathbf{2 b}$ com $\mathbf{2}$, nota-se por $\mathrm{RMN}{ }^{13} \mathrm{C}$ a presença de um sinal adicional a 193,2 ppm (C-4) com deslocamento típico de carbonila, o que confirma a oxidação do grupo $\mathrm{OH}$ em C-4, outro dado é a ausência de deslocamento correspondente à hidroxila secundária C-4 que se dá em $\delta 69,2$ para 2 . A presença da carbonila no C-4 em 2b libera a compressão estérea no grupo metila, já que agora existe um sistema $\pi$ conjugado planar entre C-1 e C-4 causando um efeito de proteção de 2 ppm na metila C-19; aspectos semelhantes foram observados em $\mathbf{3 b}$.
Os espectros de $\operatorname{COSY}{ }^{1} \mathrm{H}-{ }^{1} \mathrm{H}$ de $\mathbf{2} \mathbf{b}$ e $\mathbf{3 b}$ apresentaram grande semelhança com seus precursores $\mathbf{2}$ e $\mathbf{3}$, a exceção dos $3 \mathrm{H}(\mathrm{H}-2$, H-3 e H-4) no anel A, que não estão correlacionados em $\mathbf{2 b}$ e $\mathbf{3 b}$ (Figura 4). No espectro de DEPT de $\mathbf{2 b}$ foi observada a presença de 9 grupos $\mathrm{CH}$, sendo dois deles vinílicos (139 e 142 ppm), 5 grupos $\mathrm{CH}_{2}$ e 6 grupos $\mathrm{CH}_{3}$; notou-se também o desaparecimento do sinal correspondente ao grupo $\mathrm{CH}$ a $69 \mathrm{ppm}$ presente em $\mathbf{2}$, e que foi transformado em uma carbonila em C-4 no composto $\mathbf{2 b}$; mudança semelhante ocorreu em $\mathbf{3 b}$.

O vitanolido 2 c foi obtido através de reação de hidrogenação, tendo como catalisador de reação $\mathrm{Pd} / \mathrm{C}$. As análises para identificação de $2 \mathbf{c}$ foram feitas através de técnicas de $\mathrm{RMN}$ de ${ }^{13} \mathrm{C}$ e ${ }^{1} \mathrm{H}$ e espectrometria de massas. Inicialmente, observou-se que o espectro de massas de 2c apresentou íons importantes a $m / z$ 405(M-125)+ e $m / z, 387$ $\left(\mathrm{M}-125-\mathrm{H}_{2} \mathrm{O}\right)^{+}$, auxiliando na confirmação estrutural da substância. A análise dos espectros de $\mathrm{RMN}{ }^{1} \mathrm{H}$ e $\mathrm{COSY}{ }^{1} \mathrm{H}-{ }^{1} \mathrm{H}$ de $2 \mathbf{c}$ (Figura 4) mostrou como diferenças principais em relação ao espectro de 2: o desaparecimento de dois sinais de H-vinílicos a 6,2 e 6,8 ppm, o H-4 que em $\mathbf{2}$ aparecia como um dubleto a 3,8 ppm, apareceu como um tripleto a 3,5 ppm, correspondendo a um hidrogênio vizinho a um grupo metilênico a 2,1 ppm, o deslocamento do ${ }^{1} \mathrm{H}$ foi semelhante a dados da literatura com vitanolido apresentando a mesma composição anel A. No DEPT de 2c percebe-se claramente o desaparecimento dos carbonos vinílicos (CH) a 132,0 e 142,0 ppm. Comparando-se o espectro de $\mathrm{RMN}{ }^{13} \mathrm{C}$ de $2 \mathrm{c}$ com 2 nota-se a presença de cinco sinais na região entre 122 e 210 ppm, e não sete como em $\mathbf{2}$, devido ao desaparecimento de dois grupos $\mathrm{CH}$ vinílicos após a redução da dupla ligação C2-C3.

Os derivados obtidos visam levar a um aumento da atividade citotóxica em relação aos seus precursores $\mathbf{2}$ e 3, que já haviam sido testados anteriormente contra vários tipos de células cancerosas. ${ }^{5} \mathrm{~A}$ diminuição da atividade biológica pode ser um indicativo da importância de determinada porção da molécula, orientando transformações posteriores. A atividade citotóxica dos derivados foi avaliada contra um grupo de células cancerosas humanas. De acordo com protocolo estabelecido pelo National Cancer Institute (NCI) dos Estados Unidos ${ }^{20}$ valores de $\mathrm{ED}_{50}$ maiores que $5 \mu \mathrm{g} / \mathrm{mL}$ são considerados inativos contra células cancerosas; para uma análise em relação à potencial droga anticâncer são apresentados, na Tabela 1, os valores de $\mathrm{ED}_{50}$ para agentes antitumorais Taxol e Camptotecina, utilizados terapeuticamente. ${ }^{21,22}$ Os derivados com valores de $\mathrm{ED}_{50}$ no intervalo de $0,1-1 \mu \mathrm{g} / \mathrm{mL}$ foram considerados fortemente ativos contra células cancerosas (Tabela 1). Os resultados mais significativos foram obtidos em células de câncer de pulmão, com os derivados $\mathbf{2 c}$ e $\mathbf{3 a}$ com $\mathrm{ED}_{50}$ de 0,63 e $0,25 \mu \mathrm{g} / \mathrm{mL}$, respectivamente, com valores próximos ao da Camptotecina $\left(\mathrm{ED}_{50}=0,01 \mu \mathrm{g} / \mathrm{mL}\right)$.

Comparando-se a atividade biológica de $\mathbf{2}$ com o derivado $\mathbf{2 a}$ acetilado no C-4, percebe-se que a atividade diminui nos cânceres de mama e cólon, mas aumenta 7 vezes nas células de câncer de pulmão; comportamento semelhante foi observado quando se comparou o derivado 3a com seu precursor 3. Com relação aos derivados $\mathbf{2 b}$ e $\mathbf{2 c}$, os resultados apresentados mostram que 2c melhorou em 2 vezes a atividade para câncer de pulmão, mas a atividade caiu nos outros tipos de células. Os derivados $\mathbf{2} \mathbf{b}$ e $\mathbf{3 b}$, que têm a incorporação de uma carbonila, não apresentaram nenhum aumento na atividade nas células testadas, entretanto percebe-se, tanto para $\mathbf{2} \mathbf{b}$ como para $\mathbf{3 b}$, um decréscimo na atividade menos significativo para as células de câncer epidermoide bucal, o que mostra maior seletividade da substância neste grupo de células, podendo orientar transformações em outros centros da molécula.

A respeito dos resultados pudemos observar, para os derivados 2a e 3a, que a introdução de um grupo mais volumoso como o grupo acetato, com desaparecimento da hidroxila, que pode formar ligação 
de hidrogênio, favoreceu a melhora na atividade biológica em células de câncer de pulmão. Nos derivados $\mathbf{2} \mathbf{b}$ e $\mathbf{3 b}$ a mudança da geometria tetraédrica em C-4 para trigonal plana levou a uma diminuição significativa de atividade em relação a células cancerosas de pulmão, mostrando a importância da realização de modificações estruturais em substâncias naturais.

\section{MATERIAL SUPLEMENTAR}

Os espectros de RMN ${ }^{1} \mathrm{H}, \mathrm{COSY}, \mathrm{RMN}{ }^{13} \mathrm{C}$ e DEPT de 2, 3, 2a, 2b, 2c, 3a e 3b estão disponíveis, com acesso livre, na forma de arquivo PDF, em http://quimicanova.sbq.org.br.

\section{AGRADECIMENTOS}

Ao CNPq pela concessão de bolsa e recursos financeiros. À University of Illinois em Chicago pela realização dos testes biológicos.

\section{REFERENCIAS}

1. Braz-Filho, R.; Quim. Nova 2010, 33, 229.

2. Lavie, D.; Yarden, A.; J. Chem. Soc. 1962, 2925; Lavie, D.; Greenfield, S.; Glotter, E.; J. Chem. Soc. 1966, 1753; Glotter, E.; Nat. Prod. Rep. 1991, 8, 415 .

3. Kupchan, S. M.; Anderson, W. K.; Bollinger, P.; Doskotch, R. W.; Smith, R. M.; Renauld, J. A. S.; Schnoes, H. K.; Burlingame, A. L.; Smith, D. H.; J. Am. Chem. Soc. 1965, 87, 5805.

4. Barata, L. E. S.; Mors, W. B.; Kirson, I.; Lavie, D.; An. Acad. Bras. Cienc. 1970, 42, 401.

5. Minguzzi, S.; Barata, L. E. S.; Shin, G. S.; Chai, H. B.; Park, E. J.; Pezzuto, J. M.; Cordell, G. A.; Phytochemistry 2002, 59, 635.

6. Veras, M. L.; Bezerra, M. Z. B.; Lemos, T. L. G.; Uchoa, D. E. A.; BrazFilho, R.; Chai, H. B.; Cordell, G. A.; Pessoa, O. D. L.; J. Nat. Prod. 2004, 67, 710.

7. Veras, M. L.; Bezerra, M. Z. B.; Braz-Filho, R.; Pessoa, O. D. L.; Montenegro, R. C.; Pessoa, C.; Moraes, M. O.; Lotufo, L. V. C.; Planta Med. 2004, 67, 710.

8. Cordero, C. P.; Morantes, S. J.; Paez, A.; Rincón, J.; Aristizábal, F. A.; Fitoterapia 2009, 80, 364.

9. Manickam, M.; Awasthi, S. B.; Sinha-Bagchi, A.; Sinhá, S. C.; Ray, A. B.; Phytochemistry 1996, 41, 981; Manickam, M.; Srivastava, A.; Ray,
A. B.; Phytochemistry 1998, 47, 1427; Bratati, D.; Fitoterapia 2003, 74, 14; Bhat, B. A.; Dhar, K. L.; Puri, S. C.; Qurishi, M. A.; Khajuria, A.; Gupta, A.; Qazi, G. N.; Bioorg. Med. Chem. 2005, 13, 6672.

10. Nicotra, V. E.; Gil, R. R.; Vaccarini, C. E.; Oberti, J. C.; Burton, G.; J. Nat. Prod. 2003, 66, 1471; Nicotra, V. E.; Ramacciotti, N. S.; Gil, R. R.; Oberti, J. C.; Feresin, G. E.; Guerrero, C. A.; Baggio, R. F.; Garland, M. T.; Burton, G.; J. Nat. Prod. 2006, 69, 783; Nicotra, V. E.; Gil, R. R.; Oberti, J. C.; Burton, G.; J. Nat. Prod. 2007, 70, 808.

11. Maldonado, E.; Torres, F. R.; Martínez, M.; Pérez-Castorena, A. L.; Planta Med. 2004, 70, 59; Lan, Y. H.; Chang, F. R.; Pan, M. J.; Wu, C. C.; Wu, S. J.; Chen, S. L.; Wang, S. S.; Wu, M. J.; Wu, Y. C.; Food Chem. 2009, 116, 462; Maldonado, E.; Amador, S.; Martínez, M.; PérezCastorena, A. L.; Steroids 2010, 75, 346.

12. Choudhary, M. I.; Shahwar, D.; Parveen, Z.; Jabbar, A.; Ali, I.; Rahman, A. U.; Phytochemistry 1995, 40, 1243.

13. Benjumea, D.; Herrera, M.; Abdala, S.; Gutiérrez-Luis, J.; Quiñones, W.; Cardona, D.; Torres, F.; Echeverri, F.; J. Ethnopharmacol. 2009, 123, 351.

14. Misico, R. I.; Song, L. L.; Veleiro, A. S.; Cirigliano, A. M.; Tettamanzi, M. C.; Burton, G.; Bonetto, G. M.; Nicotra, V. E.; Silva, G. L.; Gil, R. R.; Kinghorn, A. D.; Pezzuto, J. M.; J. Nat. Prod. 2002, 65, 677.

15. Huang, C. F.; Ma, L.; Sun, L. J.; Ali, M.; Arfan, M.; Liu, J. W.; Hu, L. H.; Chem. Biodivers. 2009, 6, 1415.

16. Pomilio, A. B.; Falzoni, E. M.; Vitale, A. A.; Nat. Prod. Commun. 2008, $3,593$.

17. Silva, T. M. S.; Camara, C. A.; Freire, K. R. L.; Silva, T. G.; Agra, M. F.; Bhattacharyya, J.; J. Braz. Chem. Soc. 2008, 19, 1048.

18. Counsell, R. E.; Klimstra, P. D.; Colton, F. B.; J. Org. Chem. 1962, 27, 248.

19. Likhitwitayawuid, K.; Angerhofer, C. K.; Cordell, G. A.; Pezzuto, J. M.; J. Nat. Prod. 1993, 56, 30.

20. Ito, A.; Chai, H. B.; Shin, Y. G.; Mej, R. M.; Gao, Q.; Fairchild, C. R.; Lane, K. E.; Menendez, A. T.; Farnsworth, N. R.; Cordell, G. A.; Pezzuto, J. M.; Kinghorn, D.; Tetrahedron 2000, 56, 6401.

21. Pisha, E.; Chai, H.; Lee, I.-S.; Chagwedera, T. E.; Farnsworth, N. R.; Cordell, G. A.; Beecher, C. W. W.; Fong, H. H. S.; Kinghorn, A. D.; Brown, D. M.; Wani, M. C.; Wall, M. E.; Hieken, T. J.; Das Gupta, T. K.; Pezzuto, J. M.; Nature 1995, 1, 1046.

22. Hwang, B. Y.; Chai, H.; Kardono, L. B. S.; Riswan, S.; Farnsworth, N. R.; Cordell, G. A.; Pezzuto, J. M.; Kinghorn, A. D.; Phytochemistry 2003, 62, 197 . 


\section{DERIVADOS CITOTÓXICOS DE VITANOLIDOS ISOLADOS DAS FOLHAS DE Acnistus arborescens}

\section{Sandro Minguzzi*}

Curso de Química, Universidade Estadual de Mato Grosso do Sul, Unidade de Naviraí, Rua Emílio Máscoli, 275, 79950-000 Naviraí - MS, Brasil

\section{Lauro E. S. Barata}

Instituto de Química, Universidade Estadual de Campinas, CP 6154, 13083-970 Campinas - SP, Brasil

Geoffrey A. Cordell

Natural Products Inc., 60203 Evanston - IL, USA

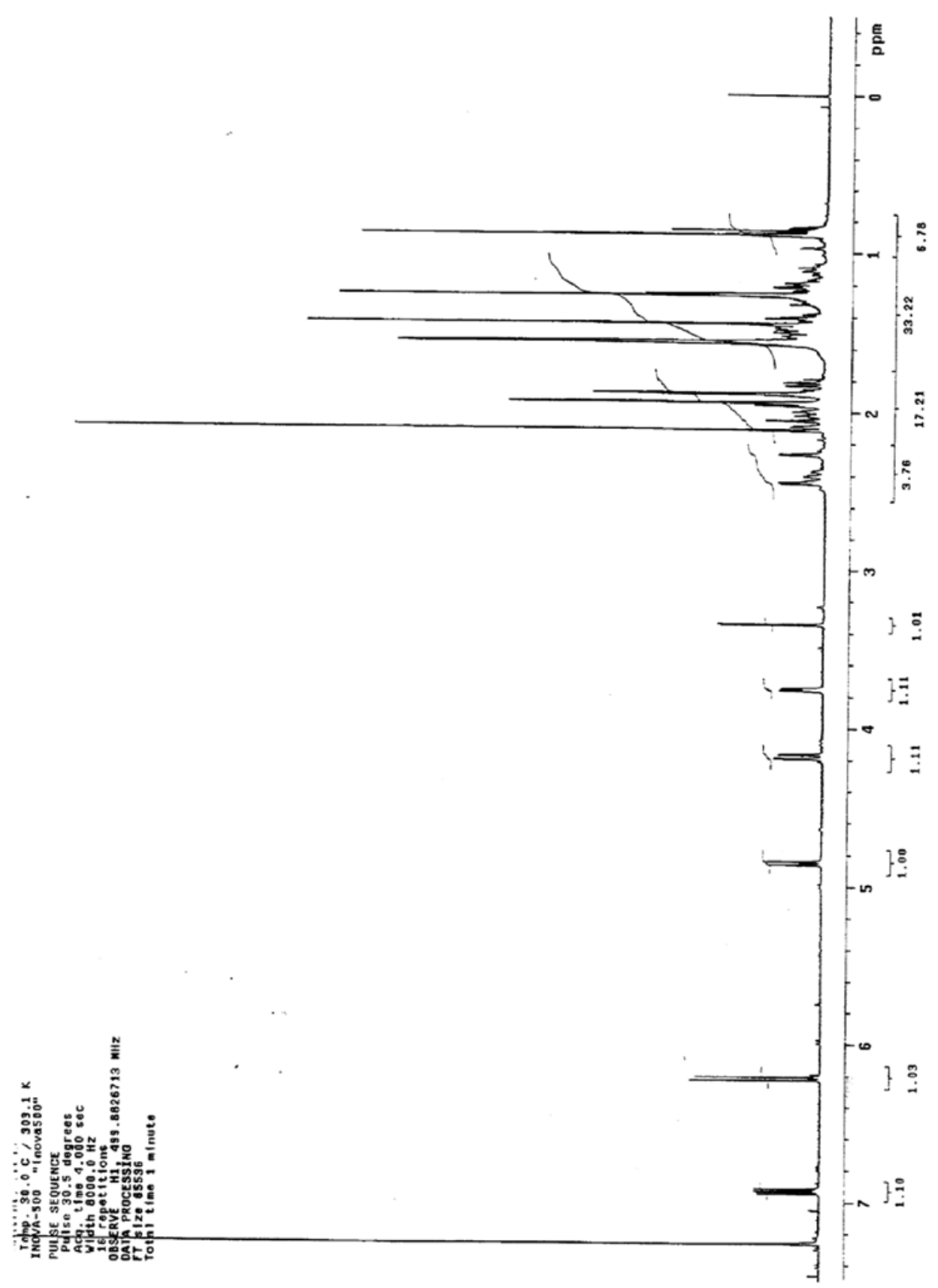

Figura 1S. Espectro de $R M N^{1} \mathrm{H}$ de 2 


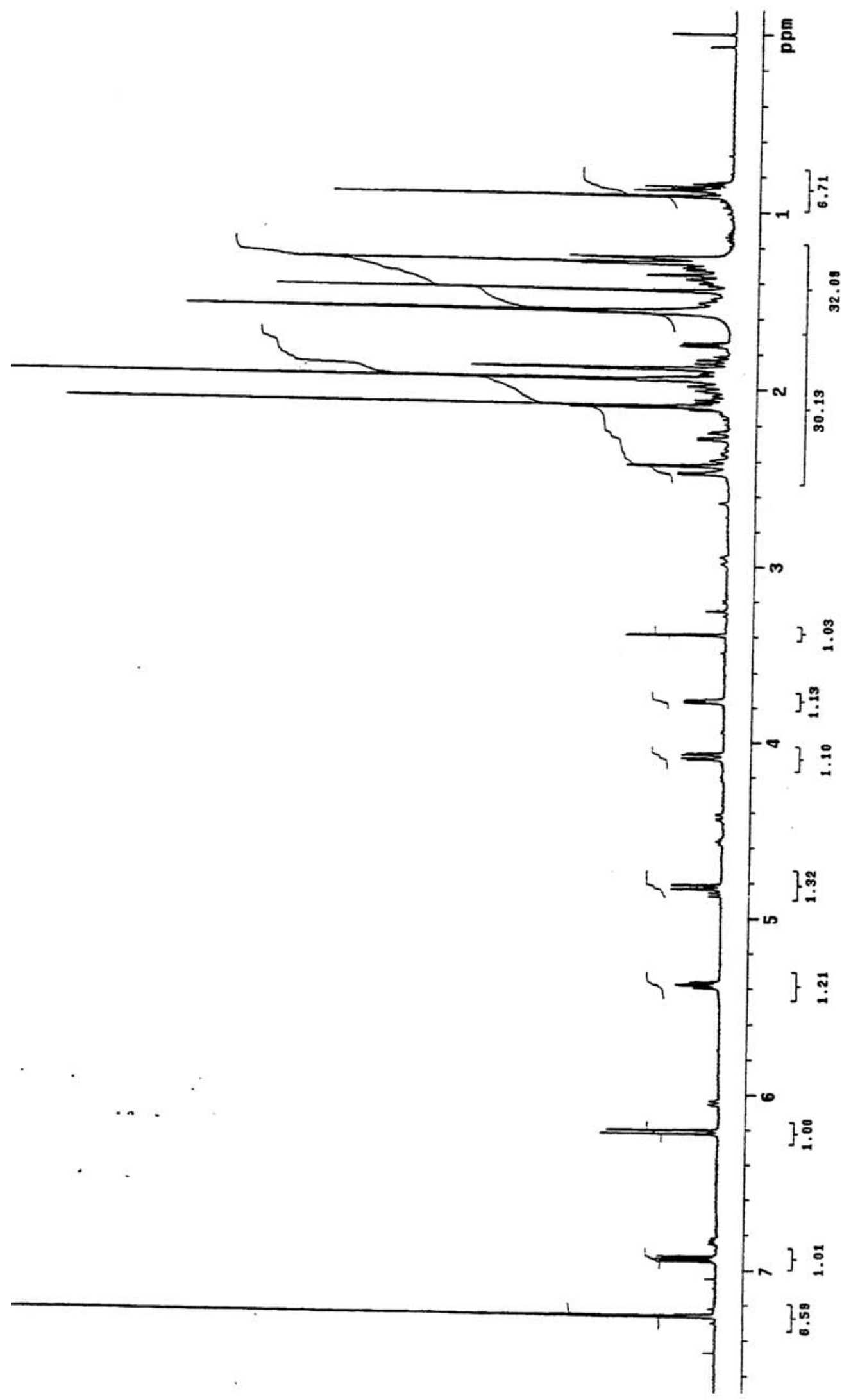

Figura 2S. Espectro de $R M N^{l} H$ de 3 


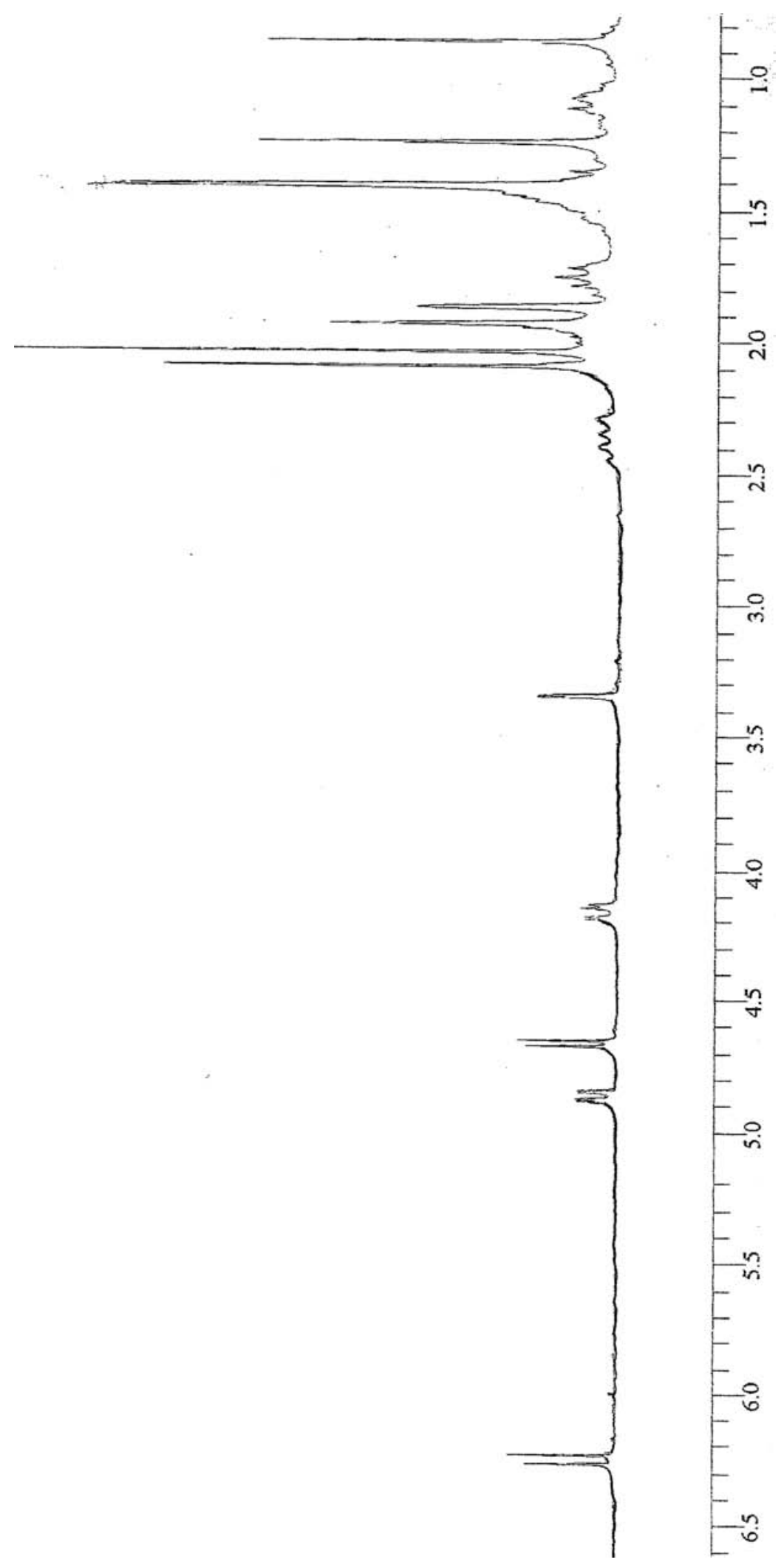

Figura 3S. Espectro de $R M N^{1} H$ de $2 \boldsymbol{a}$ 


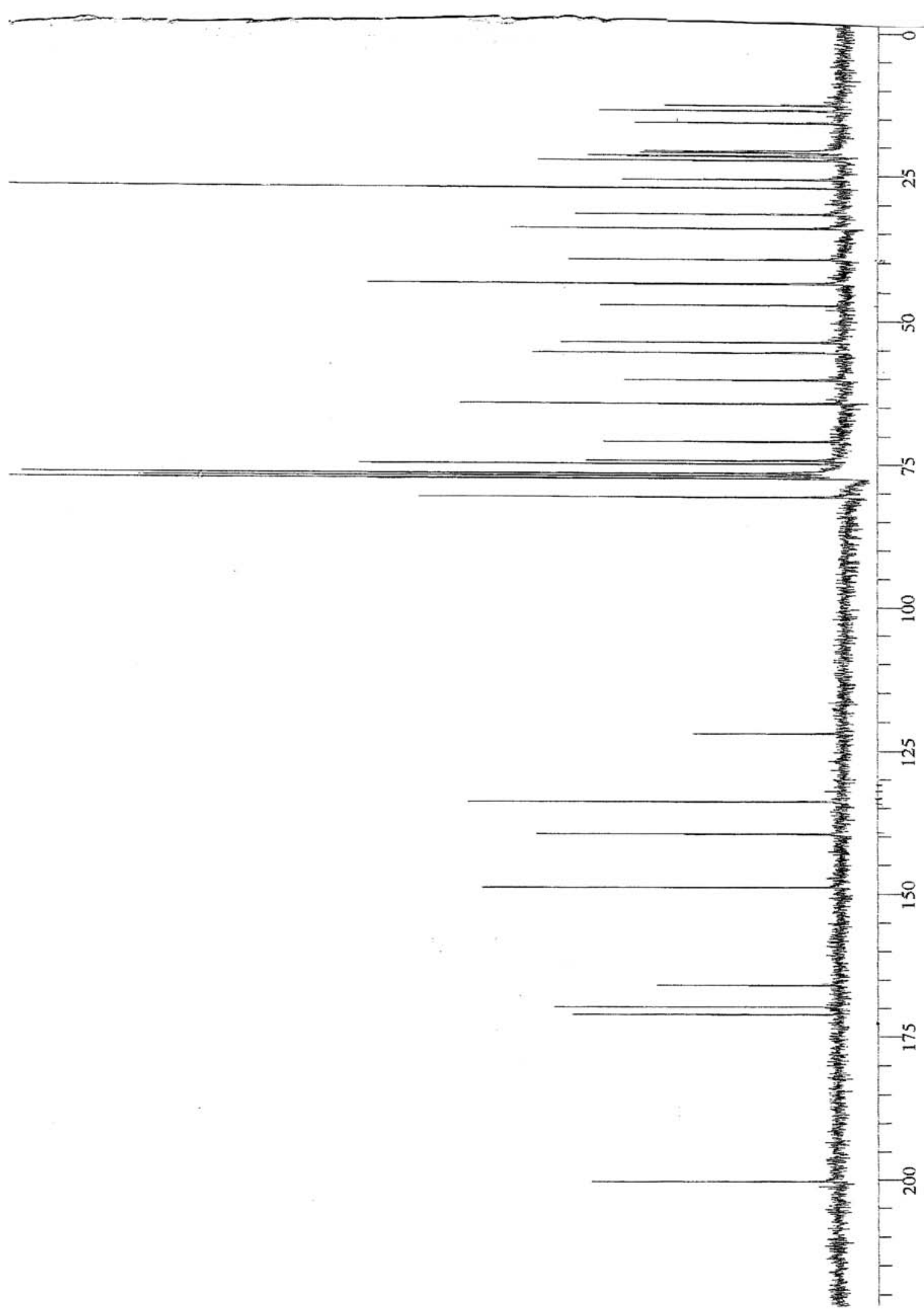

Figura 4S. Espectro de $\mathrm{RMN} d \mathrm{de}^{13} \mathrm{C}$ de $\mathbf{2 a}$ 
Vol. 34, No. 2

Derivados citotóxicos de vitanolidos isolados das folhas de Acnistus arborescens

S5
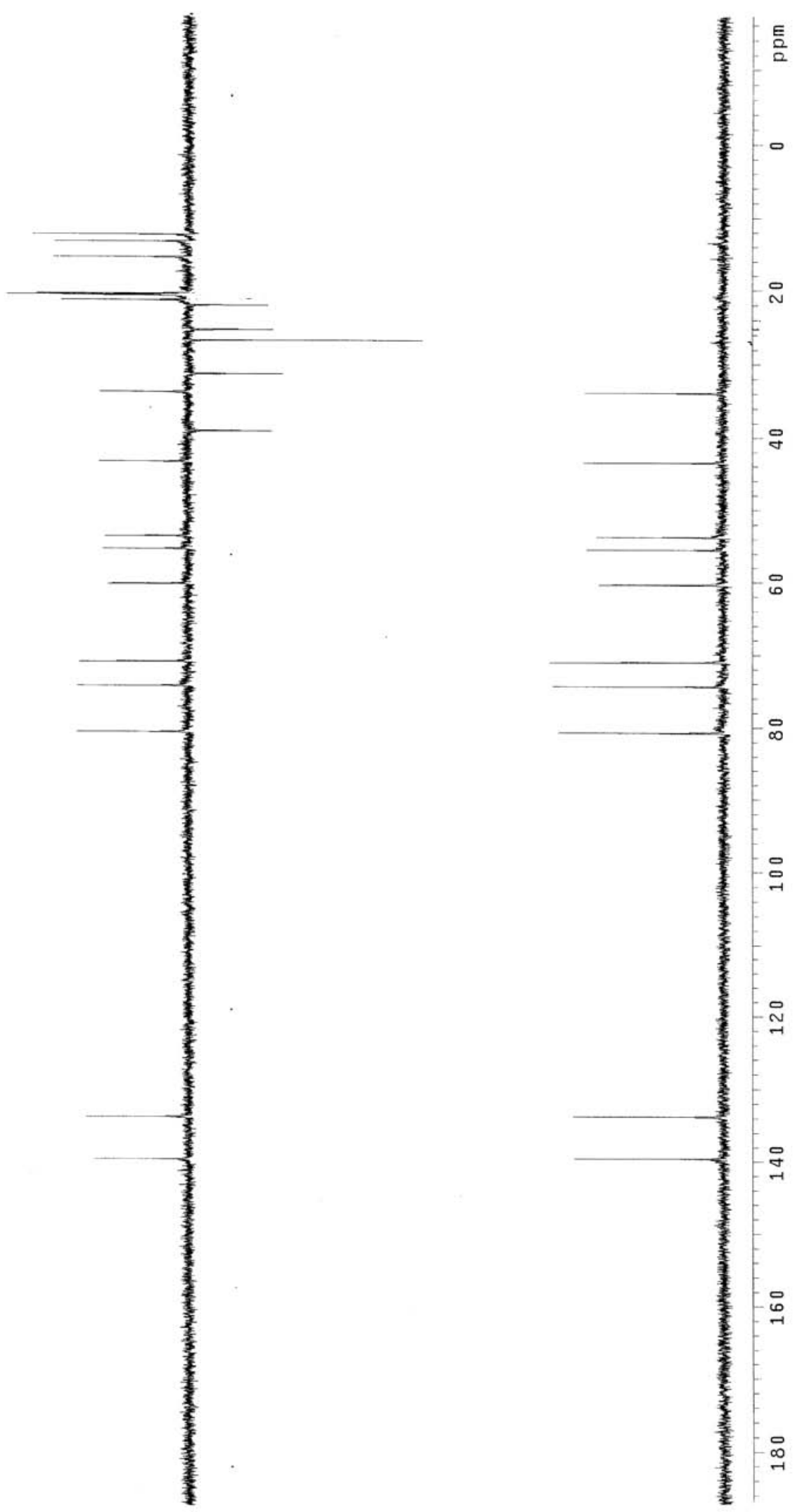

Figura 5S. Espectro DEPT de $2 a$ 


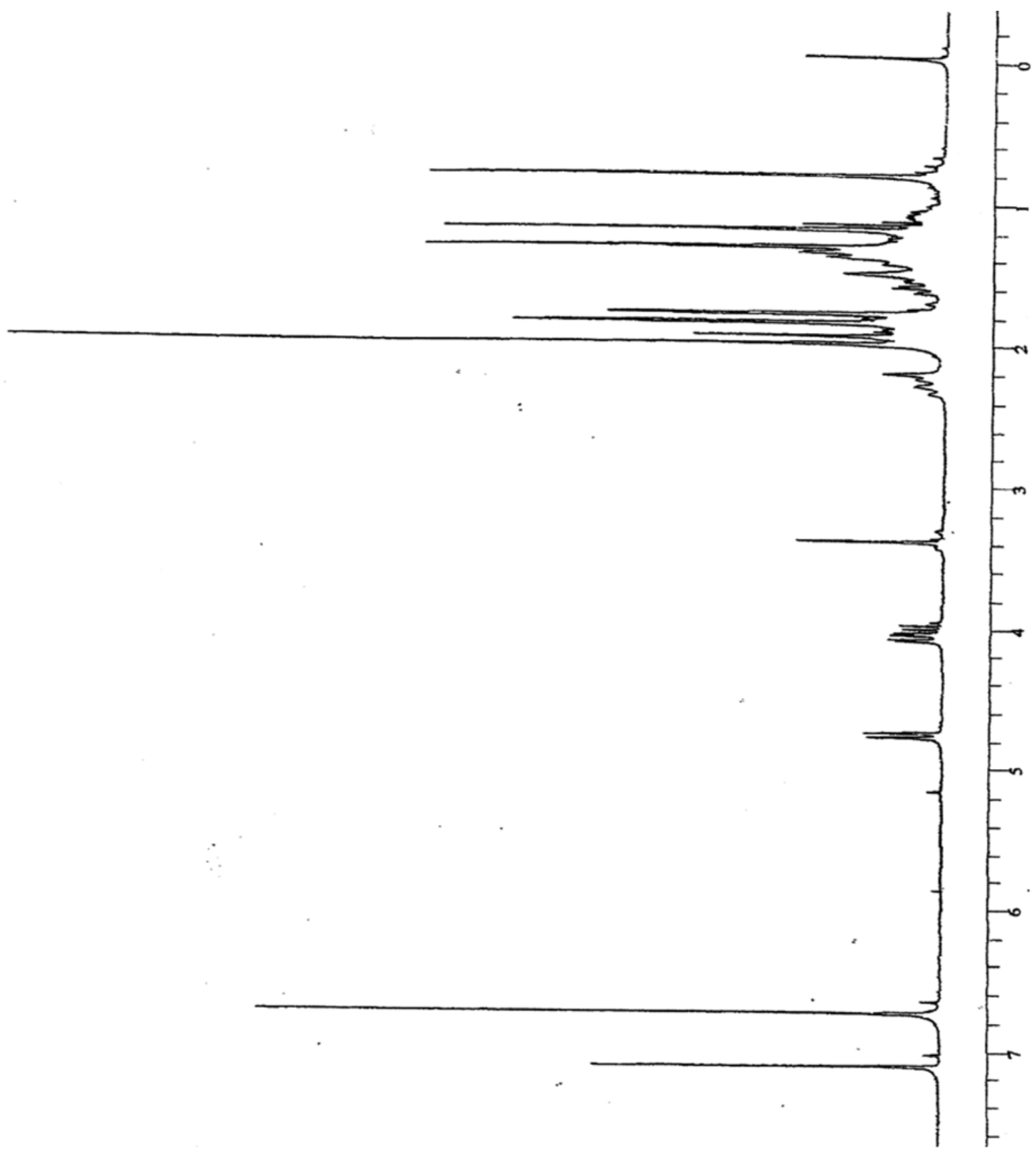

Figura 6S. Espectro de $R M N^{l} H$ de $2 \boldsymbol{b}$ 


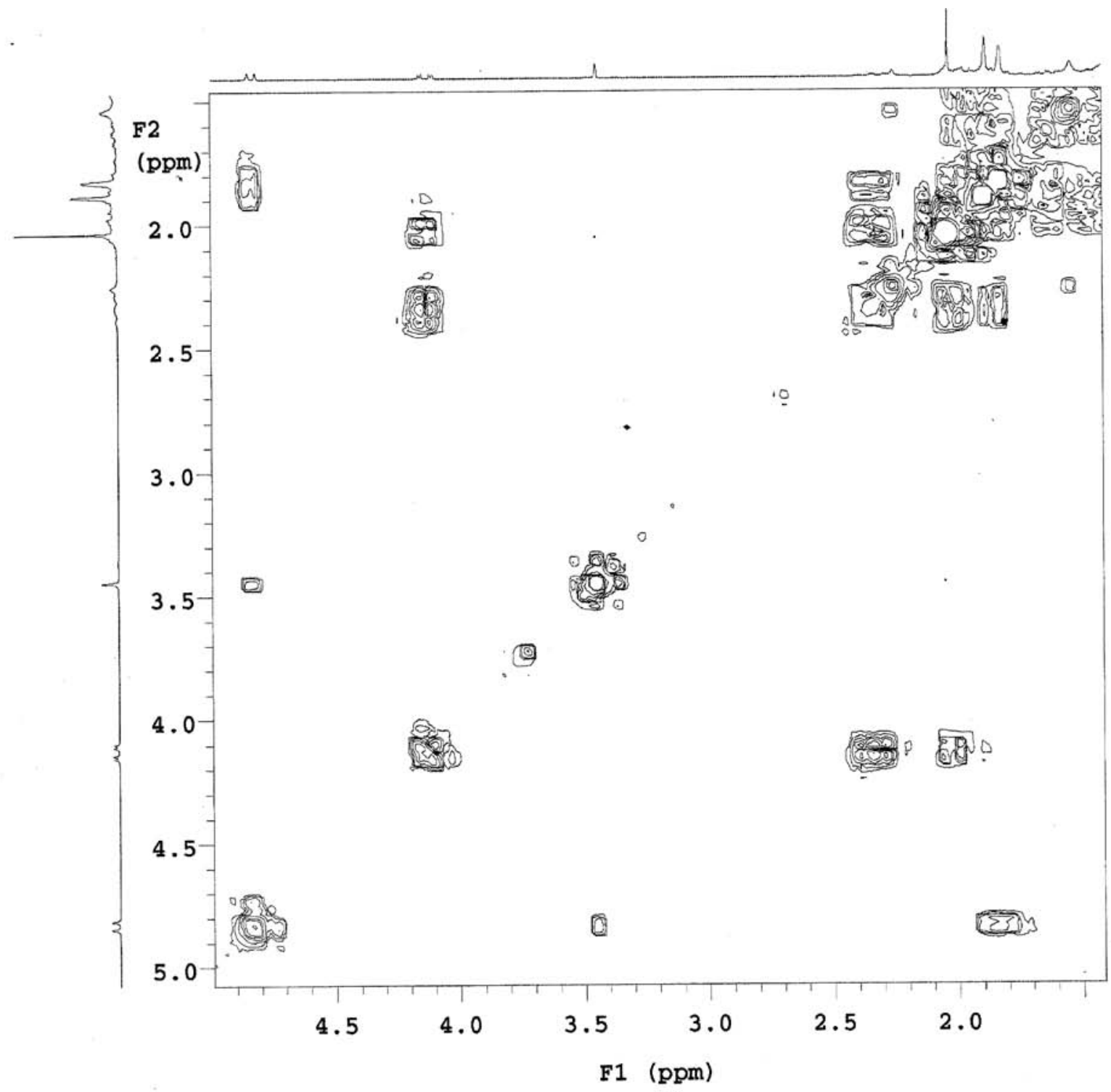

Figura 7S. Espectro $\operatorname{COSY}^{1} H-{ }^{1} H$ de $2 \boldsymbol{b}$ 


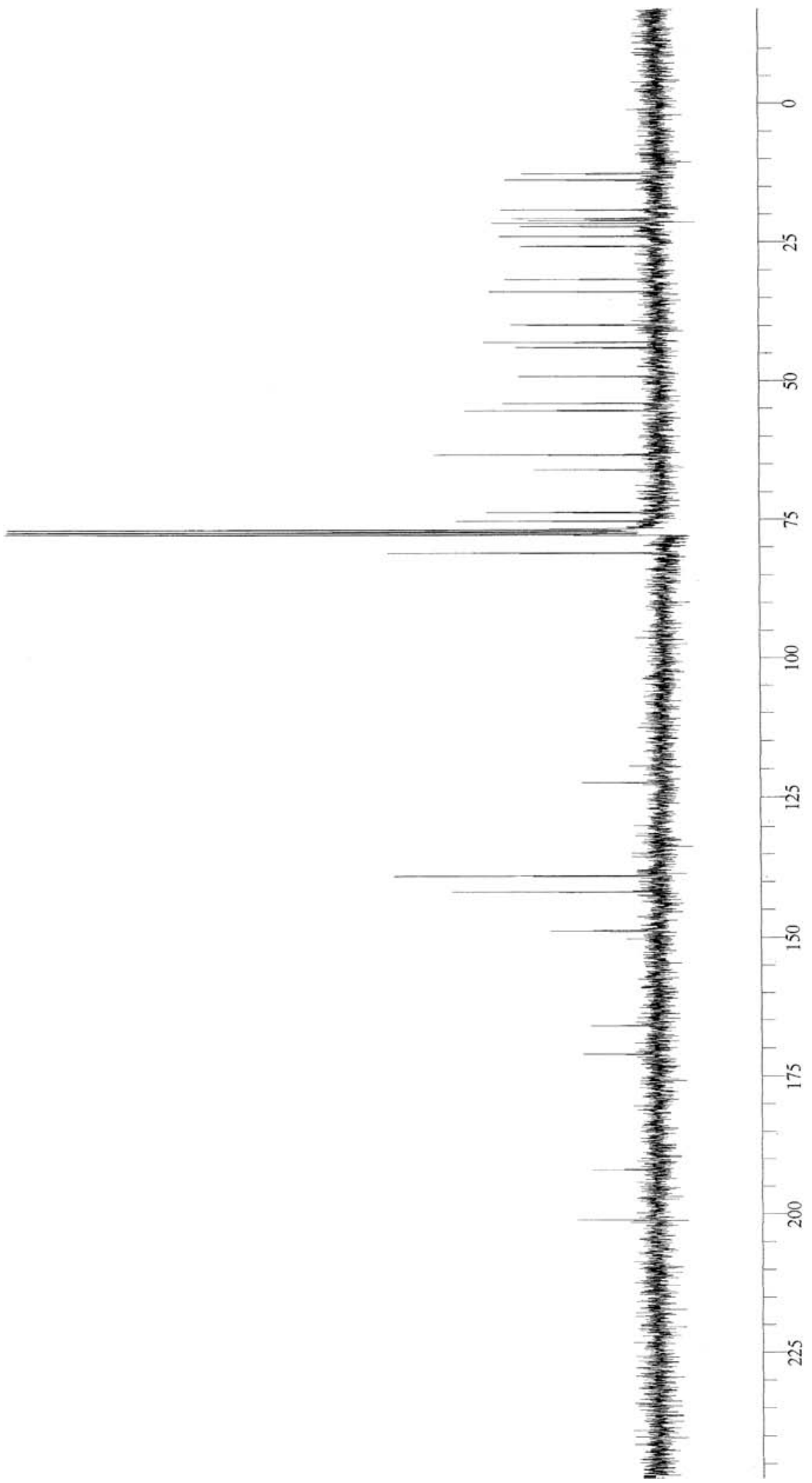

Figura 8S. Espectro de $R M N$ de ${ }^{13} C$ de $2 \boldsymbol{b}$ 


$$
11
$$




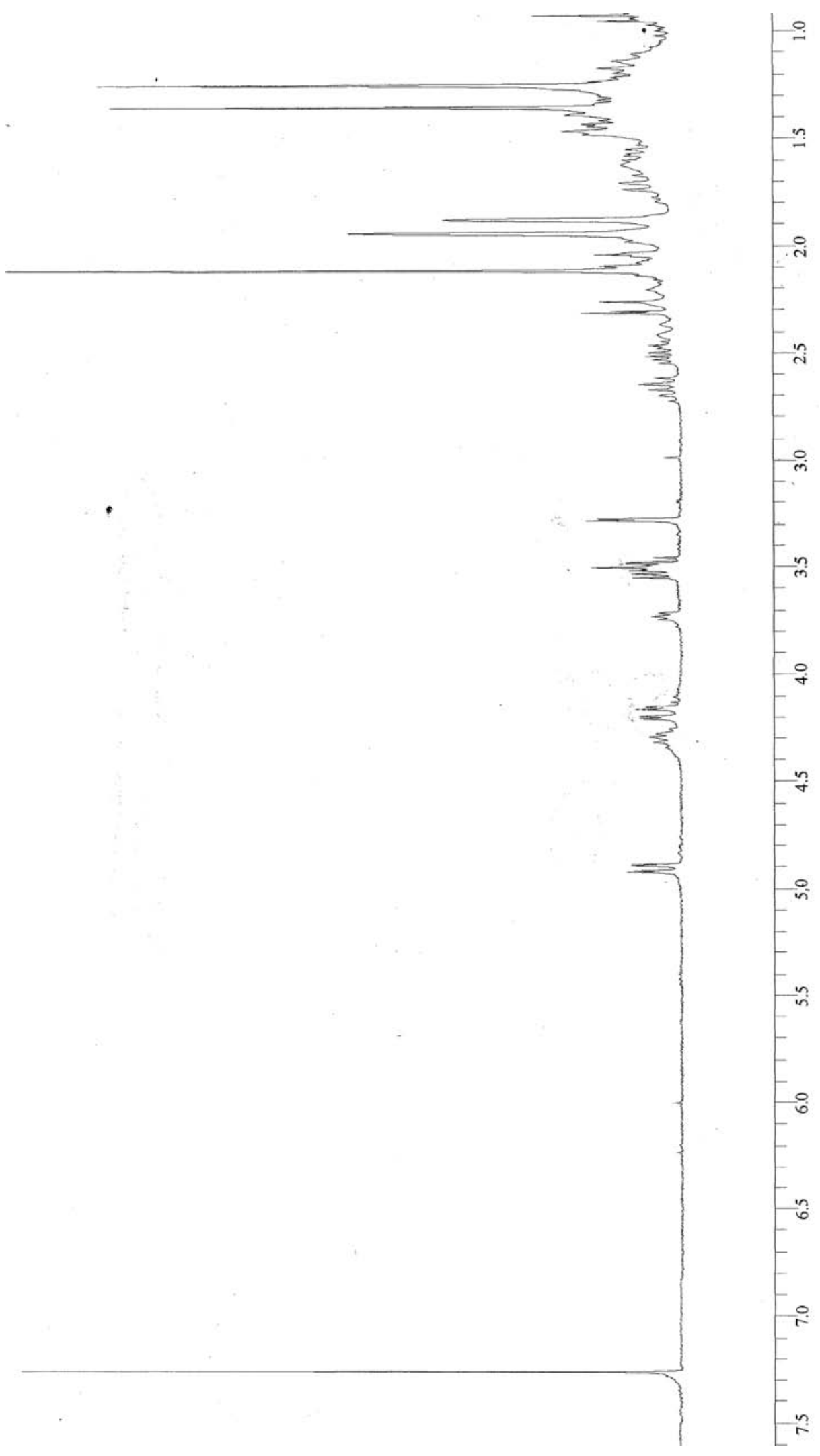




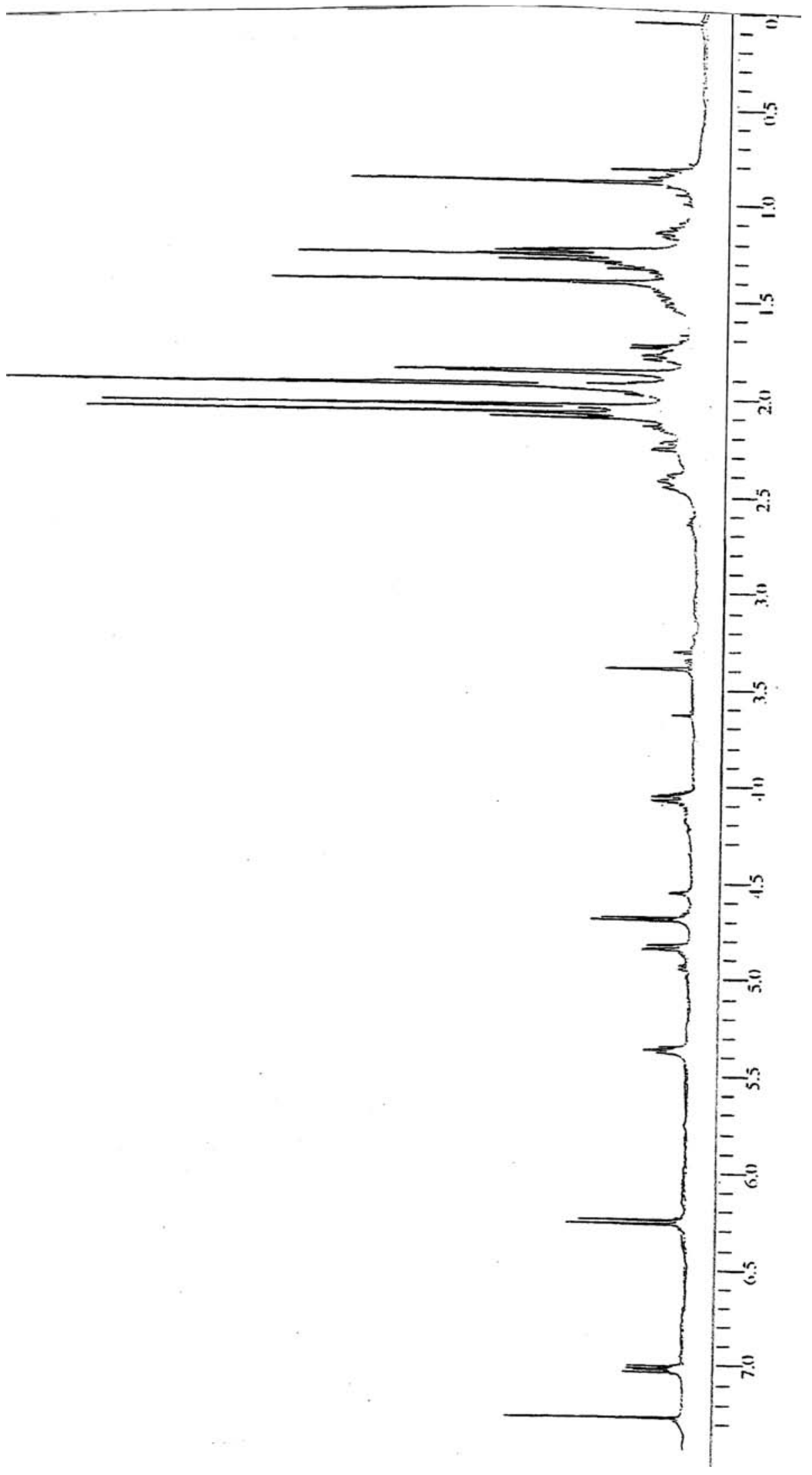




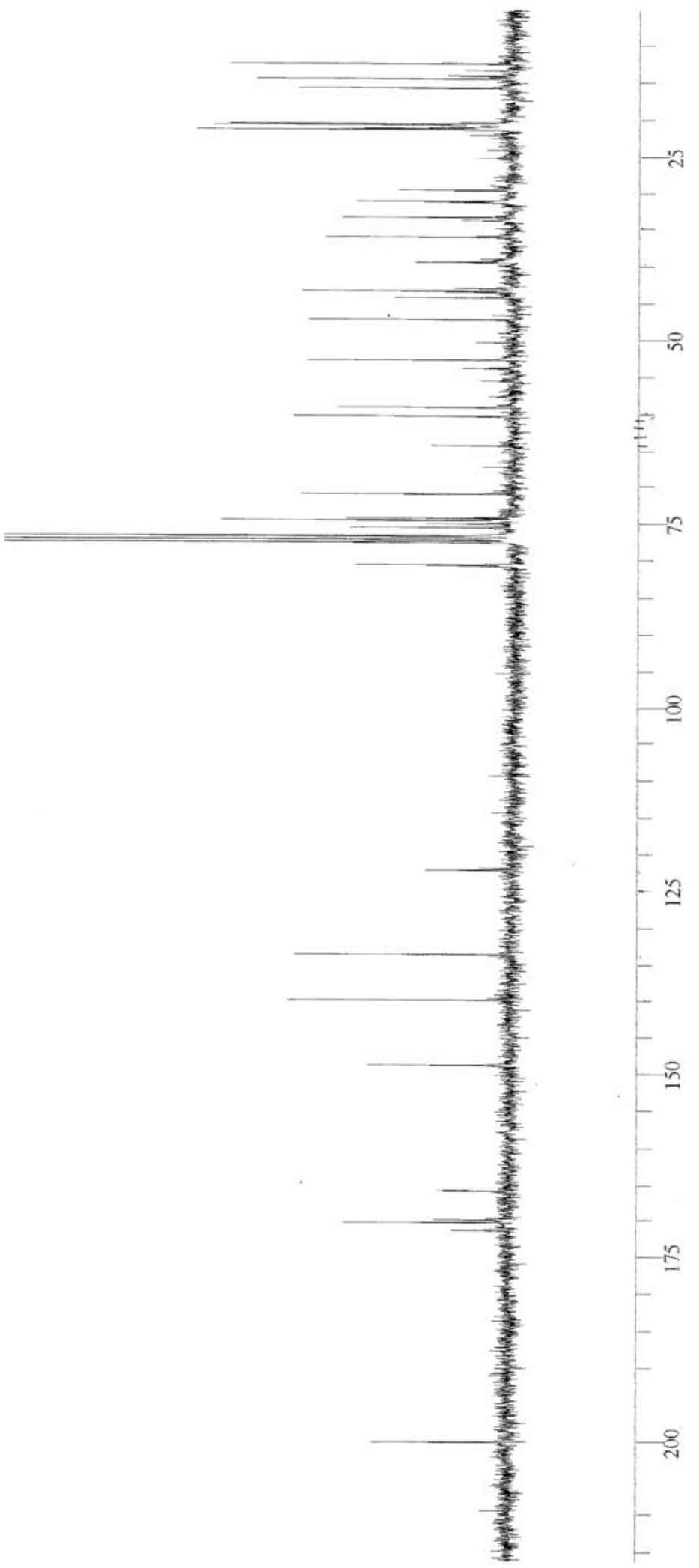

Figura 12S. Espectro de $R M N$ de ${ }^{13} \mathrm{C}$ de $\mathbf{3 a}$ 

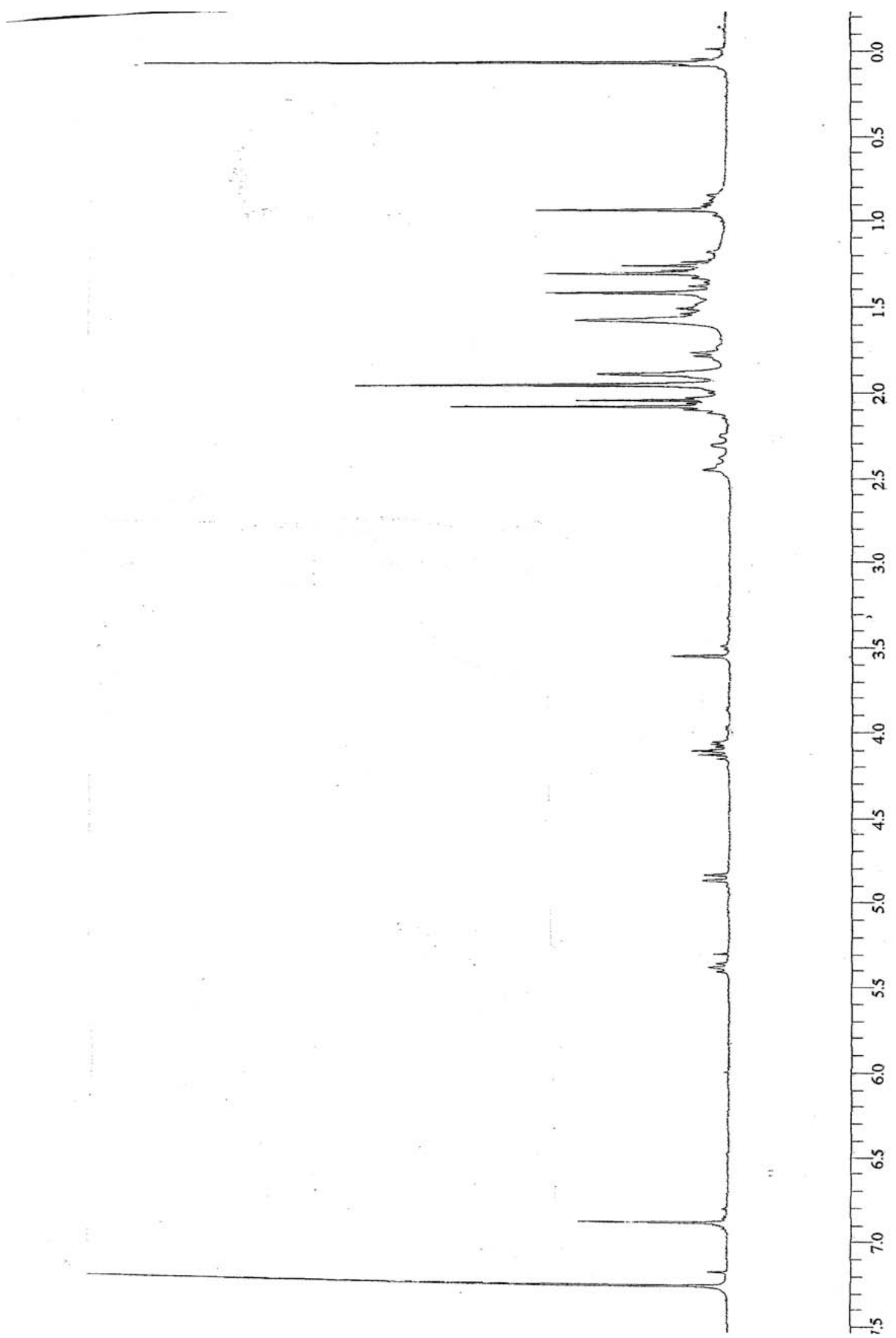

Figura 13S. Espectro de $R M N^{1} H$ de 3 b 